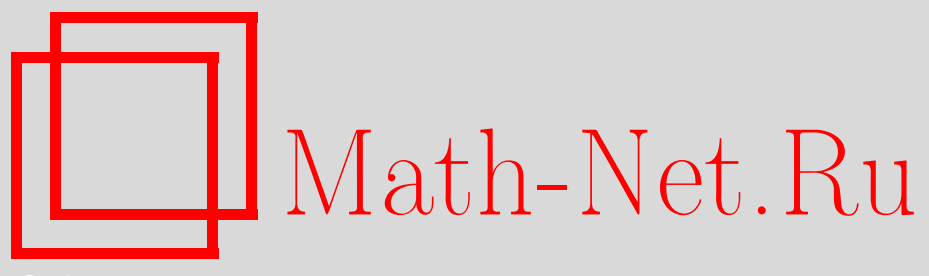

М. С. Агранович, В. М. Бухштабер, Р. С. Исмагилов, Б. С. Кашин, Б. С. Митягин, С. П. Новиков, В. А. Садовничий, А. Г. Сергеев, Я. Г. Синай, А. А. Шкаликов, Анатолий Гордеевич Костюченко (некролог), УМH, 2010, том 65, выпуск 4, 179-190

DOI: https://doi.org/10.4213/rm9369

Использование Общероссийского математического портала Math-Net.Ru подразумевает, что вы прочитали и согласны с пользовательским соглашением http://www.mathnet.ru/rus/agreement

Параметры загрузки:

IP : 3.82 .47 .9

26 апреля 2023 г., 13:12:03

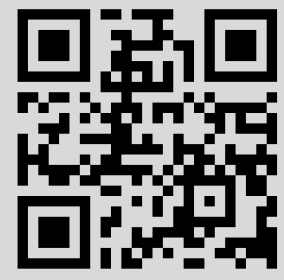




\section{Анатолий Гордеевич Костюченко}

2 апреля 2010 г. ушел из жизни профессор механико-математического факультета МГУ Анатолий Гордеевич Костюченко.

А.Г. Костюченко родился 19 апреля 1931 г. на станции Опытное Поле Синельниковского района Днепропетровской области. В 1949 г. он поступил на механико-математический факультет Киевского государственного университета. Летом 1953 г. в Киевский университет приехал Андрей Николаевич Колмогоров. Его целью было знакомство со студентами, чтобы отобрать наиболее способных для поступления в аспирантуру Московского университета. С 4-го курса, на котором учился Костюченко, рекомендован был он один.

В 1954 г., после окончания Киевского государственного университета, он поступил в аспирантуру механико-математического факультета

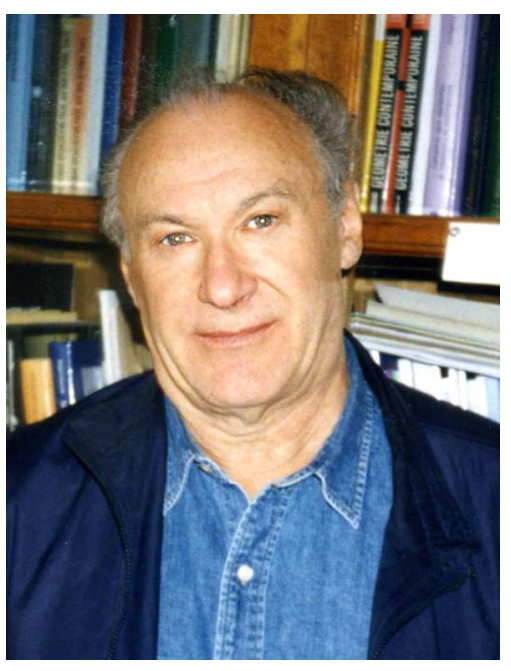
Московского университета. Его научным руководителем стал Георгий Евгеньевич Шилов. Помимо Шилова серьезное влияние на его научные интересы оказал Израиль Моисеевич Гельфанд, который в те годы работал вместе с Шиловым над первыми тремя томами известной серии монографий "Обобщенные функции”. Именно этой тематике были посвящены первые исследования Костюченко. Отметим один из важнейших его результатов, полученных в этом направлении.

Согласно определению Гельфанда-Шилова, система дифференциальных уравнений с частными производными

$$
\frac{\partial u}{\partial t}=P\left(i \frac{\partial}{\partial x}\right) u, \quad x=\left(x_{1}, \ldots, x_{n}\right) \in \mathbb{R}^{n},
$$

где $P(i \partial / \partial x)=\left\{P_{j k}(i \partial / \partial x)\right\}_{1}^{s}$ - матрица, называется регулярной, если фундаментальная матрица $Q(s, t)$ системы обыкновенных дифференциальных уравнений

$$
\frac{\partial Q}{\partial t}=P(s) Q, \quad s=\left(s_{1}, \ldots, s_{n}\right) \in \mathbb{C}^{n},
$$

есть целая функция от $s$ порядка роста $p$, причем

$$
\|Q(\sigma+i \tau, t)\| \leqslant C(t) e^{a|\tau|^{p}}(1+|\sigma|)^{l},
$$

где $C, a, p$ и $l$ - постоянные. Развив технику преобразования Фурье для быстро растущих функций, Гельфанд и Шилов доказали единственность решения задачи Коши для рассматриваемой системы в классе функций, допускающих оценку

$$
|u(x, t)| \leqslant C e^{b|x|^{p^{\prime}}}, \quad \frac{1}{p}+\frac{1}{p^{\prime}}=1 .
$$


Костюченко доказал, что существование решения задачи Коши можно гарантировать в этом же классе. Замечательно то, что для доказательства этого результата он нашел важное обобщение на случай целых функций конечного порядка известной теоремы Пэли-Винера-Шварца для преобразования Фурье функций экспоненциального типа, растущих на вещественной оси не быстрее степени.

В совместных работах Гельфанд и Костюченко получили фундаментальные результаты о разложении по собственным функциям самосопряженных операторов в функциональных гильбертовых пространствах. Эти результаты впоследствии развивались в работах многих математиков и вошли в ряд монографий. Исследованиям Гельфанда-Костюченко предшествовала одна важная задача, изученная Р. А. Александряном по предложению С. Л. Соболева. При изучении задачи Дирихле для волнового уравнения со спектральным параметром возникала некоторая динамическая система, эргодические свойства которой зависели от свойств собственных функций соответствующей спектральной задачи. Собственные функции оказались обобщенными (не принадлежащими основному пространству), что почти всегда случается в случае непрерывного спектра. Александряну удалось доказать их полноту в некотором пространстве функционалов. В 1954 г. на Международном математическом конгрессе в Амстердаме Гельфанд поставил задачу о существовании полной системы обобщенных собственных функций инфинитезимального оператора

$$
A=i \sum_{k=1}^{n} \frac{\partial X_{k}\left(x_{1}, \ldots, x_{n}\right)}{\partial x_{k}}, \quad x \in \mathbb{R}^{n},
$$

любой классической динамической системы

$$
\frac{d X_{i}}{d t}=X_{i}\left(x_{1}, \ldots, x_{n}\right), \quad i=1, \ldots, n, \quad \operatorname{div}\left(X_{1}, \ldots, X_{n}\right)=0,
$$

при условии, что этот инфинитезимальный оператор самосопряжен в $L_{2}(\mathbb{R})$.

Вскоре Гельфанд и Костюченко решили задачу о полноте и разложении по собственным функциям для произвольного самосопряженного оператора в гильбертовом пространстве. Им принадлежит замечательная конструкция тройки $\Phi \subset H \subset \Phi^{\prime}$, где $H$ - основное пространство, в котором действует оператор, $\Phi$ плотно вложено в $H$, а $\Phi^{\prime}$ - пространство линейных непрерывных функционалов на $\Phi$, двойственное к $\Phi$ относительно скалярного произведения в $H$. Конструкция пространства $\Phi$ осуществлялась с наделением его некоторыми свойствами, в результате чего Гельфанд и Костюченко фактически пришли к понятию ядерного пространства. Интересно отметить, что независимо и другим путем к понятию ядерного пространства пришел также А. Гротендик, который и ввел этот термин. Далее, с помощью спектральной функции $E_{\lambda}$ и порождающего вектора $e$ Гельфанд и Костюченко строили меру $\sigma(\lambda)=\left(E_{\lambda} e, e\right)$ и доказывали (это является наиболее трудным моментом), что существует производная

$$
\frac{d E_{\lambda} e}{d \sigma(\lambda)}=\chi_{\lambda},
$$

понимаемая в слабом смысле, т.е. как функционал на $\Phi$. Система функционалов $\chi_{\lambda} \in \Phi^{\prime}$ образует систему обобщенных собственных функций рассматриваемого оператора (в случае обыкновенных или эллиптических дифференциальных операторов функционалы $\chi_{\lambda}$ являются обычными решениями соответствующих уравнений на собственные значения). Для всех $\varphi \in \Phi$ была доказана справедливость разложения

$$
\varphi=\int_{-\infty}^{+\infty}\left(\chi_{\lambda}, \varphi\right) \chi_{\lambda} d \sigma(\lambda)
$$

и равенства Парсеваля

$$
\|\varphi\|^{2}=\int_{-\infty}^{+\infty}\left|\left(\chi_{\lambda}, \varphi\right)\right|^{2} d \sigma(\lambda)
$$


Впоследствии существенное развитие теория Гельфанда-Костюченко получила в известной монографии Ю. М. Березанского.

В 1959 г. А. Г. Костюченко и Б. С. Митягин заметили, что общая теорема Гельфанда-Костюченко о разложении по собственным функциям в применении к системам коммутирующих самосопряженных операторов дает возможность получить представления для многомерных положительно определенных ядер и функционалов. Ранее подобные представления с привлечением метода направляющих функционалов в одномерном случае получил М.Г. Крейн. Эта идея нашла воплощение в серии работ, выполненных Костюченко и Митягиным. За эти работы им была присуждена премия Московского математического общества. Полученные новые теоремы о представлении многомерных положительно определенных ядер и функционалов в конечном итоге позволили решить трудную задачу многомерной проблемы моментов: установить ее определенность, или единственность порождающей меры, при специальных условиях на моментную последовательность (нужно отметить, что в отличие от одномерного случая многомерная проблема моментов не всегда разрешима). Для $n=2$ независимо и другим методом похожие результаты для проблемы моментов получил А. Девинатц (Devinatz). Основные трудности в предложенном Костюченко и Митягиным подходе сводились к решению следующей проблемы: когда симметрические коммутирующие операторы $A_{1}, \ldots, A_{n}$, заданные на общей области определения, допускают расширения, определяющие систему коммутирующих самосопряженных операторов на общей области определения? Костюченко и Митягин нашли эффективные условия, гарантирующие положительное решение этой проблемы. Их работы по этой тематике были впоследствии существенно развиты Р. С. Исмагиловым и Г. И. Эскиным, а также Ю. М. Березанским. Впоследствии эти исследования получили развитие и в работах зарубежных математиков в связи с аксиоматическим построением квантовой теории поля.

Большой интерес вызвали работы Березанского и Костюченко об оценках собственных функций эллиптических операторов. Одним из известных их результатов по этой теме является следующий. Пусть эллиптический оператор в $\mathbb{R}^{n}$ таков, что ядро его резольвенты $K(x, \xi, \lambda)$ при некотором $\lambda$ допускает оценку

$$
\int_{-\infty}^{+\infty}|K(x, \xi, \lambda)|^{2} d \xi \leqslant C
$$

где постоянная $C$ не зависит от $x \in \mathbb{R}^{n}$. Тогда собственные функции этого оператора растут не быстрее степени, а именно, при любом $\varepsilon>0$

$$
|\varphi(x, \lambda)| \leqslant C(\varepsilon)|x|^{n / 2+\varepsilon} .
$$

Костюченко был в числе первых математиков, кто смог получить общие результаты по спектральной теории дифференциальных операторов высших порядков, как обыкновенных, так и в частных производных. В частности, в 60-е годы он впервые получил асимптотику функции распределения собственных значений эллиптического оператора высшего порядка в $\mathbb{R}^{n}$. До его работ асимптотические формулы плотности спектра были известны только для оператора Шрёдингера и некоторых других классов дифференциальных операторов второго порядка. Эти формулы до работ Костюченко были получены как вариационным методом, так и с помощью предложенного Т. Карлеманом и развитого Е. Титчмаршем тауберова метода. Костюченко изучил эллиптический оператор вида

$$
L(x, D)=\sum_{|\alpha|=2 m} a_{\alpha}(x) D^{\alpha}+L_{1}(x, D)+q(x),
$$

где $L_{1}(x, D)$ - оператор $(2 m-1)$-го порядка, рост коэффициентов которого подчинен росту функции $q(x)$. С этим оператором он связал задачу Коши для параболического 
уравнения

$$
\frac{\partial u(x, t)}{\partial t}=-L(x, D) u(x, t), \quad u(x, 0)=\varphi(x) .
$$

В случае дискретного спектра функция распределения собственных значений $N(\lambda)$ оператора $L(x, D)$ оказывается связанной с функцией Грина $G(t, x, \xi)$ рассматриваемой задачи Коши равенством

$$
\int G(t, x, x) d x=\int e^{-t \lambda} d N(\lambda) .
$$

Исследовав асимптотику функции Грина $G$ методом параметрикса и использовав тауберовы теоремы для преобразований Лапласа, Костюченко получил формулу

$$
N(\lambda) \sim(2 \pi)^{-n} \int_{\mathbb{R}^{n}} \Phi(x)(\lambda-q(x))^{n /(2 m)} d x,
$$

где

$$
\Phi(x)=\operatorname{mes}\left\{\xi \in \mathbb{R}^{n} \mid \sum_{|\alpha|=2 m} a_{\alpha}(x) \xi^{\alpha} \leqslant \lambda\right\} .
$$

Аналогичные формулы он получил для эллиптических систем и некоторых классов неэллиптических операторов. Этим же методом он получил асимптотические формулы взвешенного следа операторов. Изложенный метод параболического уравнения часто называют методом Костюченко. В последующие годы результаты об асимптотиках спектра дифференциальных операторов обобщались, усиливались и уточнялись многими авторами (в частности, в работах М.Ш. Бирмана, Д. Г. Васильева, В.Я. Иврия, М. О. Отелбаева, Г. А. Розенблюма, Ю. Г. Сафарова, М. З. Соломяка, М. А. Шубина и др.)

При изучении асимптотики спектральной функции $Q(\lambda, x, y)$ - ядра спектрального семейства $E_{\lambda}$ для самосопряженного обыкновенного дифференциального оператора

$$
P=(-1)^{m} \frac{d^{2 m}}{d x^{2 m}}+\sum_{j=0}^{2 m-2} p_{j}(t) \frac{d^{j}}{d x^{j}}, \quad x \in \mathbb{R},
$$

Костюченко смог корректно определить корень $2 m$-й степени из оператора $P$, хотя в то время теории псевдодифференциальных операторов еще не было. С помощью полученного операторного корня ему удалось установить связь между тауберовой теоремой Ганелиуса для преобразования Лапласа и тауберовой теоремой Левитана-Марченко для преобразования Фурье. На этом пути он получил асимптотику

$$
Q(\lambda, x, \xi)=\frac{\sin \lambda^{1 /(2 m)}(x-y)}{\pi(x-y)}+o(1),
$$

где $|o(1)| \rightarrow 0$ при $\lambda \rightarrow \infty$ равномерно на любом компакте по переменным $x$ и $\xi$. Здесь важно, что остаток имеет вид $o(1)$, а не $O(1)$ - это обстоятельство значительно расширяет область приложений полученной асимптотики. Например, это важно в задачах разложения по собственным функциям, вычисления регуляризованных следов и др. Этот результат Костюченко неулучшаем и не выводится из более поздних результатов Хёрмандера. Впоследствии результаты Костюченко о спектральных асимптотиках эллиптических и обыкновенных дифференциальных операторов получили развитие в работах многих математиков, в особенности его учеников. В 80-е годы он продолжил работу над этой темой в серии совместных работ с К. Х. Бойматовым. Как одно из важных достижений следует отметить результаты этой серии, в которых в получающихся асимптотиках участвуют не только главный символ и потенциал, но и "средние" члены, вносящие равноценный вклад с "крайними". Кроме того, в их работах детально рассмотрен случай вырождающихся коэффициентов, потребовавший преодоления значительных технических трудностей. 
В конце 60-х годов у Костюченко возникает серьезный интерес к теории несамосопряженных операторов, операторных пучков и оператор-функций. Он изучает результаты М. В. Келдыша о полноте собственных функций полиномиальных операторных пучков, а также результаты Л. С. Понтрягина, М. Г. Крейна и Г. К. Лангера по теории операторов в пространстве с индефинитной метрикой. Его привлекает глубина идей, заложенных в работах этих авторов, и тесная связь этой теории с приложениями. В 1975 г. Костюченко совместно с М. Б. Оразовым получил важное дополнение к теореме Крейна-Лангера о факторизации квадратичного самосопряженного пучка. Согласно этой теореме, квадратичный пучок операторов с ограниченным оператором $B=B^{*}$ и компактным $C>0$ допускает представление

$$
L(\lambda):=\lambda^{2}+\lambda B+C=\left(\lambda-Z_{1}\right)(\lambda-Z),
$$

где спектр оператора $Z$ содержится в замкнутой верхней полуплоскости. Важно знать ответ на вопрос: как описать вещественный спектр оператора $Z$ и его структуру? Костюченко и Оразов получили полный ответ на этот вопрос. Они изучили еще одну важную задачу: как движутся вещественные собственные значения $\lambda_{j}(\omega)$ пучка $L(\lambda)-\omega R$, где $R>0$ - ограниченный оператор, при изменении вещественного параметра $\omega$; как расщепляется резонансное собственное значение, т. е. собственное значение $\lambda_{j}\left(\omega_{0}\right)$, которому при $\omega=\omega_{0}$ отвечает жорданова клетка или набор таких клеток?

До начала 80-х годов в литературе изучались в основном только пучки ограниченных операторов. Но конкретные задачи, возникающие в теории упругости и гидромеханике, приводили к изучению пучков неограниченных операторов. Конечно, имелись некоторые процедуры редукции пучков с неограниченными операторными коэффициентами к пучкам с ограниченными операторами, но при этом терялась существенная информация. Теория факторизации пучков с неограниченными операторными коэффициентами получила развитие, начиная с работы А.Г. Костюченко и А. А. Шкаликова, вышедшей в 1983 г. Наряду с другими изученными в этой работе задачами, авторы выделили класс эллиптических пучков вида

$$
L(\lambda)=\lambda^{2} F+\lambda G+H,
$$

где оператор $F$ равномерно положителен и ограничен, $H=H^{*}$ полуограничен, $G \subset G^{*}$ и $\mathfrak{D}(G) \supset \mathfrak{D}(H)$. Были найдены эффективно проверяемые условия, гарантирующие возможность представления

$$
L(\lambda)=\left(\lambda-Z_{1}\right)(\lambda-Z),
$$

где оператор $Z$ таков, что: 1$)$ спектр $Z$ лежит в замкнутой верхней полуплоскости, а на вещественной оси состоит из собственных значений первого рода (при наличии жордановых клеток описание вещественного спектра $Z$ усложняется); 2 ) $i Z$ является генератором голоморфной полугруппы в некоторой шкале пространств, построенных по оператору $H$. В доказательстве этой теоремы существенно использовались как геометрический, так и аналитический методы теории несамосопряженных операторов. Эта теорема имела важные приложения. Она дала возможность доказать существование и единственность решения так называемой усеченной задачи Коши на полупрямой для уравнения $L(i d / d x) u(x)=0$. С ее помощью дано обоснование гипотезы Мандельштама о существовании и единственности решений уравнений электродинамики в полуцилиндре с условиями излучения на бесконечности; она позволила дать полное решение задачи Рэлея об отражении волны от периодической поверхности (ранее решение этой задачи было известно только в плоском случае при отсутствии резонансных частот). Эти приложения также были реализованы в работах Костюченко и Шкаликова.

В 1943 г. в отчетах Математического института им. В. А. Стеклова появилась работа С.Л. Соболева, в которой изучалась задача об устойчивости движения волчка с полостью, заполненной идеальной жидкостью. Соболев обратил внимание на 
то, что в случае идеальной жидкости задача сводилась к изучению некоторого оператора, который симметризуется в индефинитном скалярном произведении с одним отрицательным квадратом. Соболев провел первые исследования таких операторов и заинтересовал этой тематикой Л. С. Понтрягина, который в 1944 г. написал работу, заложившую основы теории операторов в пространствах с индефинитной метрикой. Сама задача Соболева об устойчивости для волчка как с идеальной, так и с вязкой жидкостью, впоследствии изучалась многими математиками и механиками, в частности, Н. Н. Моисеевым, Дж. Ральстоном, В. В. Румянцевым, Ф. В. Черноусько. Были получены некоторые достаточные условия как устойчивости, так и неустойчивости. Реализовав в современной форме идеи начальной работы Соболева и развив теорию диссипативных операторов в пространстве с индефинитной метрикой, А. Г. Костюченко, А. А. Шкаликова и М. Ю. Юркин в 1998 г. дали полное решение задачи Соболева с вязкой жидкостью, указав необходимые и достаточные условия устойчивости задачи, причем не только конечномерной “твердотельной” ее части (в предшествующих работах задача устойчивости изучалась только для “твердотельной" компоненты), но и всей задачи в целом. За работы по теории несамосопряженных операторов и ее приложениям Костюченко и Шкаликов в 2001 г. были удостоены Ломоносовской премии первой степени.

В дальнейшем Костюченко вновь вернулся к изучению проблемы моментов и связанной с ней теории якобиевых матриц и ортогональных многочленов. В 1998-2001 гг. совместно с К. А. Мирзоевым он опубликовал по этой тематике серию работ. В частности, Костюченко и Мирзоев нашли критерий, а также различные достаточные условия неопределенности (т.е. неоднозначной разрешимости) матричной проблемы моментов. Интересно отметить, что некоторые из их условий являются новыми и в скалярном случае.

В 2006-2009 гг. А. Г. Костюченко и Р. С. Исмагилов выполнили серию работ об асимптотиках спектра векторного оператора Штурма-Лиувилля. В этих работах впервые были получены спектральные асимптотики, зависящие не только от собственных значений матрицы-потенциала, но также от "скорости вращения" его собственных векторов. Этот новый эффект был изучен также для неполуограниченных операторов. В работе [77] Исмагилов и Костюченко изучили характер изменения спектра операторов Штурма-Лиувилля при возмущении потенциала произвольной измеримой ограниченной функцией. В этой же работе впервые дано подробное (и достаточно простое) изложение уже упомянутой теоремы Костюченко об асимптотике фундаментального решения задачи Коши для параболического уравнения.

А.Г. Костюченко обладал замечательным даром - даром Учителя. Умение видеть и ставить новые проблемы, атмосфера увлеченности на семинарах, которыми он руководил, всегда привлекали к Анатолию Гордеевичу большое число учеников. Более 60 из них защитили кандидатские диссертации. Многие впоследствии стали докторами наук. Среди них: Р. С. Исмагилов, В.А. Садовничий, М. М. Гехтман, М. О. Отелбаев, К. Х. Бойматов, Г. В. Радзиевский, А.Г. Сергеев, Я. Т. Султанаев, Г. И. Исаев, Б. А. Искендеров, М. Б. Оразов, А. А. Шкаликов, А. М. Гомилко, О. Велиев, Е. Д. Нурсултанов, А. К. Фрагела, В. В. Власов, С. А. Степин.

В течение многих лет А. Г. Костюченко являлся руководителем известной школы по теории операторов, отмеченной грантом Президента Российской Федерации для ведущих научных школ России.

Много энергии А. Г. Костюченко отдавал общественной работе на механико-математическом факультете, научно-организационной и издательской деятельности. Более 30 лет он являлся членом диссертационного совета механико-математического факультета, в 1990-1998 гг. избирался вице-президентом Московского математического общества. На протяжении многих лет он был членом редколлегий журналов "Успехи математических наук", "Функциональный анализ и его приложения", "Фундаментальная и прикладная математика". 
Друзья и коллеги Анатолия Гордеевича Костюченко, вся российская математика понесли с его кончиной невосполнимую утрату.

М. С. Агранович, В. М. Бухштабер, Р. С. Исмагилов, Б. С. Кашин, Б. С. Митягин, С.П. Новиков, В.А. Садовничий, А.Г. Сергеев, Я.Г. Синай, А.А. Шкаликов

\section{Список научных трудов А. Г. Костюченко}

[1] "О связи между строением $(n-1)$-мерной поверхности и ее главными кривизнами", УМН, 8:5 (1953), 161-164.

[2] “Об одной теореме Н. К. Бари”, УМН, 8:5 (1953), 165-166 (совм. с А. В. Скороходом).

[3] "О решении задачи Коши для регулярных систем линейных уравнений в частных производных", УМН, 9:3 (1954), 141-148 (совм. с Г.Е. Шиловым); англ. пер.: "On the solution of Cauchy's problem for regular systems of linear partial differential equations", Amer. Math. Soc. Transl. Ser. 2, 5 (1957), 275-283 (with G. E. Shilov).

[4] "О задаче Коши для линейной системы уравнений в частных производных с дифференциальными операторами Штурма-Лиувилля", Докл. АН CCCP, 98:1 (1954), 17-20.

[5] О теореме Лиувилля, Студенческие работы университета, 16, Киев, 1955.

[6] "О теореме единственности решения задачи Коши и смешанной задачи для некоторых типов систем линейных уравнений в частных производных", Докл. АН CCCP, 103:1 (1955), 13-16.

[7] "Разложение по собственным функциям дифференциальных и других операторов", Докл. АН СССР, 103:3 (1955), 349-352 (совм. с И. М. Гельфандом).

[8] "О поведении собственных функций самосопряженных операторов", Докл. АН СССР, 114:2 (1957), 249-251.

[9] "О спектральных свойствах самосопряженных эллиптических операторов", Докл. AH CCCP, 115:1 (1957), 34-37.

[10] О собственных функииях самосопряжсенных операторов, Дис. ... канд. физ.-матем. наук, МГУ, М., 1957.

[11] "О некоторых задачах теории дифференциальных уравнений с постоянными коэффициентами”, Сб. задач по теории функиий комплексного переменного, Гостехиздат, М., 1958, 79-84 (совм. с Г.Е. Шиловым).

[12] "О принципе предельной амплитуды", Вестн. Моск. ун-та. Сер. 1. Матем., мех., астр., физ., хим., 1959, № 5, 153-163 (совм. с Г. И. Бассом).

[13] "О положительно определенных функционалах на ядерных пространствах", Докл. АН СССР, 131 (1960), 13-16 (совм. с Б. С. Митягиным); англ. пер.: "Positive definite functionals on nuclear spaces", Soviet Math. Dokl., 1 (1960), 177-180 (with B.S. Mityagin).

[14] "Многомерная проблема моментов", Докл. АН СССР, 131:6 (1960), 1249-1252 (совм. с Б. С. Митягиным); англ. пер.: "The multi-dimensional problem of moments", Soviet Math. Dokl., 1 (1960), 415-419 (with B. S. Mityagin).

[15] "Положительно определенные функционалы на ядерных пространствах", Tp. MMO, 9 (1960), 283-316 (совм. с Б. С. Митягиным); англ. пер.: "Positive-definite functionals on nuclear spaces", Amer. Math. Soc. Transl. Ser. 2,93 (1970), 1-44 (with B. S. Mityagin).

[16] "Оценка резольвент сингулярных эллиптических операторов", Докл. АН CCCP, 132:1 (1960), 32-35; англ. пер.: "An estimate of the resolvents of singular elliptic operators", Soviet Math. Dokl., 1 (1960), 470-473. 
[17] “Задача Коши уравнений Соболева-Гальперна”, Тр. МMO, 10 (1961), 273-284 (совм. с Г. И. Эскиным).

[18] "Асимптотическое распределение собственных значений эллиптических операторов", Докл. АН СССР, 158:1 (1964), 41-44; англ. пер.: "Asymptotic distribution of the eigenvalues of elliptic operators", Soviet Math. Dokl., 5 (1964), 1171-1175.

[19] "Распределение собственных значений для сингулярных дифференциальных операторов", Докл. АН СССР, 168:1 (1966), 21-24; англ. пер.: "Distribution of eigenvalues of singular differential operators", Soviet Math. Dokl., 7 (1966), 584-587.

[20] "Асимптотика спектральной функции сингулярного оператора порядка $2 \mathrm{~m}$ ", Докл. АН СССР, 168:2 (1966), 276-279; англ. пер.: "Asymptotic behavior of the spectral function of a singular differential operator of order $2 m$ ", Soviet Math. Dokl., 7 (1966), 632-635.

[21] О некоторых спектралъных свойствах дифференциальных операторов, Дис. ... докт. физ.-матем. наук, МГУ, М., 1966.

[22] "О комплексных собственных значениях одного несамосопряженного оператора", Дифферени. уравнения, 3:3 (1967), 509-513 (совм. с М. М. Гехтманом); англ. пер.: "On the complex eigenvalues of a non-self-adjoint operator", Differ. Equ., 3 (1967), 259-260 (with M. M. Gekhtman).

[23] "Об асимптотическом поведении собственных значений операторной задачи Штурма-Лиувилля", Функи. анализ и его прил., 1:1 (1967), 86-96 (совм. с Б. М. Левитаном); англ. пер.: "Asymptotic behavior of the eigenvalues of the Sturm-Liouville operator problem", Funct. Anal. Appl., 1:1 (1967), 75-83 (with B. M. Levitan).

[24] "О некоторых спектральных свойствах дифференциальных операторов", Матем. заметки, 1:3 (1967), 365-378; англ. пер.: "Some spectral properties of differential operators", Math. Notes, 1 (1967), 242-250.

[25] “Асимптотическое поведение спектральной функции самосопряженных эллиптических операторов”, 4-я летняя математическая школа (Кацивели, 1966), Наукова думка, Киев, 1968, 42-117.

[26] "Некоторые вопросы спектральной теории для уравнений в частных производных", Дифференциальные уравнения в частных производных, Наука, М., 1970, 3-35 (совм. с Р. А. Александряном, Ю. М. Березанским, В.А.Ильиным); англ. пер.: "Some questions in spectral theory for partial differential equations", Amer. Math. Soc. Transl. Ser. 2, 105 (1976), 1-53 (with R. A. Aleksandryan, Yu. M. Berezanskij, V. A. Il'in).

[27] “О плотности спектра оператора Штурма-Лиувилля”, УМН, 28:2 (1973), 227-228 (совм. с В. П. Белогрудем).

[28] "О равномерной сходимости спектральных распределений", Функи. анализ и его прил., 7:2 (1973), 32-42 (совм. с Б. С. Митягиным); англ. пер.: "Uniform convergence of spectral resolutions", Funct. Anal. Appl., 7:2 (1973), 113-121 (with B. S. Mityagin).

[29] "О суммировании методом Абеля $n$-кратных разложений", Сиб. матем. журн., 15:4 (1974), 855-870 (совм. с Г. В. Радзиевским); англ. пер.: "The summability by Abel's method of $n$-tuple expansions", Siberian Math. J., 15 (1974), 607-619 (with G. V. Radzievskij).

[30] "О некоторых свойствах корней самосопряженного квадратичного пучка", Функи. анализ и его прил., 9:4 (1975), 28-40 (совм. с М.Б. Оразовым); англ. пер.: "Certain properties of the roots of a self-adjoint quadratic pencil", Funct. Anal. Appl., 9:4 (1975), 295-305 (with M. B. Orazov). 
[31] "Распределение собственных значений эллиптического оператора во всем пространстве", Тр. сем. им. И. Г. Петровского, 2 (1976), 113-143 (совм. с К. Х. Бойматовым).

[32] "О полноте корневых векторов некоторых самосопряженных квадратичных пучков”, Функи. анализ и его прил., 11:4 (1977), 85-87 (совм. с М.Б. Оразовым); англ. пер.: "The completeness of root vectors in certain self-adjoint quadratic bundles", Funct. Anal. Appl., 11:4 (1977), 317-319 (with M. B. Orazov).

[33] "Асимптотическое поведение средних Рисса спектральной функции эллиптического оператора”, Докл. АН СССР, 241:3 (1978), 517-520 (совм. с К. Х. Бойматовым); англ. пер.: "Asymptotic behavior of Riesz means of the spectral function of an elliptic operator", Soviet Math. Dokl., 19:4 (1978), 873-876 (with K. Kh. Bojmatov).

[34] "О суммируемости разложений по собственным функциям дифференциальных операторов и операторов свертки", Функи. анализ и его прил., 12:4 (1978), 24-40 (совм. с А. А. Шкаликовым); англ. пер.: "Summability of eigenfunction expansions of differential operators and convolution operators", Funct. Anal. Appl., 12:4 (1978), 262-276 (with A. A. Shkalikov).

[35] "Об одной теореме локализации”, Труды конферениии по асимптотическим методам, Алма-Ата, 1979 (совм. с К. Х. Бойматовым).

[36] Распределение собственных значений. Самосопряженные обыкновенные дифберенциальные операторы, Наука, М., 1979, 400 с. (совм. с И. С. Саргсяном).

[37] "Задача о колебаниях упругого полуцилиндра и связанные с ней самосопряженные квадратичные пучки", Тр. сем. им. И. Г. Петровского, 6 (1981), 97-146 (совм. с М. Б. Оразовым); англ. пер.: "The problem of oscillations of an elastic half cylinder and related selfadjoint quadratic pencils", J. Soviet Math., 33 (1986), 1025-1065 (with M. B. Orazov).

[38] "Самосопряженные квадратичные пучки операторов и эллиптические задачи", Функи. анализ и его прил., 17:2 (1983), 38-61 (совм. с А. А. Шкаликовым); англ. пер.: "Self-adjoint quadratic operator pencils and elliptic problems", Funct. Anal. Appl., 17:2 (1983), 109-128 (with A. A. Shkalikov).

[39] "К теории самосопряженных квадратичных пучков операторов", Вестн. Моск. ун-та. Сер. 1. Матем., мех., 1983, №6, 40-51 (совм. с А. А. Шкаликовым); англ. пер.: "On the theory of selfadjoint quadratic operator pencils", Moscow Univ. Math. Bull., 38:6 (1983), 44-58 (with A. A. Shkalikov).

[40] "К спектральной теории квадратичных пучков операторов", УМН, 39:4 (1984), 106-107 (совм. с А. А. Шкаликовым).

[41] “Асимптотические свойства спектра эллиптических операторов", Tруды VIII республиканской конференции, Алма-Ата, 1984.

[42] "Распределение собственных значений уравнения $A u=\lambda B u$ во всем пространстве”, Докл. АН СССР, 277:6 (1984), 1292-1295 (совм. с К. Х. Бойматовым); англ. пер.: "Distribution of the eigenvalues of the equation $A u=\lambda B u$ in the whole space", Soviet Math. Dokl., 30:1 (1984), 245-248 (with K. Kh. Bojmatov).

[43] "Спектральная асимптотика эллиптического оператоа в ограниченной области", Функи. анализ и его прил., 19:2 (1985), 67-69 (совм. с К. Х. Бойматовым); англ. пер.: "A spectral asymptotics of an elliptic operator in a bounded domain", Funct. Anal. Appl., 19:2 (1985), 136-138 (with K. Kh. Boimatov).

[44] "О влиянии младших коэффициентов эллиптических дифференциальных операторов на спектральную функцию", Вестн. Моск. ун-та. Сер. 1. Матем., мех., 1986, № 2, 15-23 (совм. с К.Х. Бойматовым); англ. пер.: "The effect of lowest coefficients of an elliptic differential operator on the spectral function", Moscow Univ. Math. Bull., 41:2 (1986), 17-25 (with K. Kh. Boimatov). 
[45] "О единственности решения задачи Коши для параболических уравнений", Вестн. Моск. ун-та. Сер. 1. Матем., мех., 1988, № 4, 25-32 (совм. с К. Х. Бойматовым); англ. пер.: "Uniqueness of the solution of the Cauchy problem for second-order parabolic equations with increasing coefficients", Moscow Univ. Math. Bull., 43:4 (1988), 29-38 (with K. Kh. Boimatov).

[46] "Распределение собственных значений эллиптических операторов в конечной области", Тр. сем. им. И.Г. Петровского, 13 (1988), 3-18 (совм. с К. Х. Бойматовым); англ. пер.: "The distribution of the eigenvalues of an elliptic operator in a bounded domain", J. Soviet Math., 50:1 (1990), 1337-1350 (with K. Kh. Boimatov).

[47] "О собственных значениях уравнения $A u=\lambda B u$ на компактном многообразии без края", Функи. анализ и его прил., 23:1 (1989), 63-64 (совм. с К. Х. Бойматовым); англ. пер.: "Eigenvalues of the equation $A u=\lambda B u$ on a compact manifold without boundary", Funct. Anal. Appl., 23:1 (1989), 52-53 (with K. Kh. Boimatov).

[48] "Спектральная асимптотика несамосопряженных эллиптических систем дифференциальных операторов в ограниченных областях", Функи. анализ и его прил., 24:1 (1990), 64-66 (совм. с К. Х. Бойматовым); англ. пер.: "Spectral asymptotics of non-self-adjoint elliptic systems of differential operators in bounded domains", Funct. Anal. Appl., 24:1 (1990), 54-57 (with K. Kh. Boimatov).

[49] "Спектральная асимптотика полиномиальных пучков дифференциальных операторов на компактном многообразии без края", Функи. анализ и его прил., 24:2 (1990), 76-78 (совм. с К. Х. Бойматовым); англ. пер.: "Spectral asymptotics of polynomial pencils of differential operators on a compact manifold without boundary", Funct. Anal. Appl., 24:2 (1990), 146-148 (with K. Kh. Boimatov).

[50] "Спектральная асимптотика полиномиальных пучков дифференциальных операторов", Докл. АН СССР, 313:5 (1990), 1036-1040 (совм. с К. Х. Бойматовым); англ. пер.: "Spectral asymptotics of polynomial pencils of differential operators", Soviet Math. Dokl., 42:1 (1991), 120-124 (with K. Kh. Boimatov).

[51] "Распределение собственных значений несамосопряженных дифференциальных операторов 2-го порядка", Вестн. Моск. ун-та. Сер. 1. Матем., мех., 1990, № 3, 24-31 (совм. с К.Х. Бойматовым); англ. пер.: "Distribution of eigenvalues of second-order nonselfadjoint differential operators", Moscow Univ. Math. Bull., 45:3 (1990), 26-32 (with K. Kh. Boimatov).

[52] "Спектральная асимптотика полиномиальных пучков дифференциальных операторов", Матем. заметки, 47:3 (1990), 129-130 (совм. с К. Х. Бойматовым).

[53] "Спектральная асимптотика несамосопряженных эллиптических систем дифференциальных операторов в ограниченных областях", Матем. сб., 181:12 (1990), 1678-1693 (совм. с К. Х. Бойматовым); англ. пер.: "Spectral asymptotics of nonselfadjoint elliptic systems of differential operators in bounded domains", Math. USSR-Sb., 71:2 (1992), 517-531 (with K. Kh. Boimatov).

[54] "О формуле следа для полиномиальных операторных пучков и ее приложениях", Функи. анализ и его прил., 25:4 (1991), 90-92 (совм. с К. Х. Бойматовым); англ. пер.: "Trace formula for polynomial operator pencils and its applications", Funct. Anal. Appl., 25:4 (1991), 306-308 (with K. Kh. Boimatov).

[55] "Спектральная асимптотика полиномиальных пучков дифференциальных операторов в ограниченных областях", Функи. анализ и его прил., 25:1 (1991), 7-20 (совм. с К. Х. Бойматовым); англ. пер.: "Spectral asymptotics of polynomial pencils of differential operators in bounded domains", Funct. Anal. Appl., 25:1 (1991), 5-16 (with K. Kh. Boimatov).

[56] "Ecuaciones Canónicas con Coeficientes Periódicos y Operadores en Espacios con Metrica Indefinite", Conferencias didactas, CINVESTAV, 1991. 
[57] "Self-adjoint quadratic operator pencils and elliptic problems", Trans. Confer. Math. Phys. and Diff. Equat., Morelia, Mexico, 1993.

[58] "Diffraction on a periodic surface for Helmholz's equation", Proc. Mex. Math. Soc., 48:2 (1993).

[59] "Oscillation of an elastic tube conveying liquid", Proc. Mex. Math. Soc., 49:1 (1994) (with F. Galaz-Fontes).

[60] "Oscillation of an elastic tube conveying liquid in presense of friction", Proc. Mex. Math. Soc., 49:3 (1994) (with F. Galaz-Fontes).

[61] "Критерий вполне неопределенности трехчленных рекуррентных соотношений", УМH, 51:6 (1996), 207-208 (совм. с К. А. Мирзоевым); англ. пер.: "A criterion for the complete indeterminacy of three-term recurrence relations", Russian Math. Surveys, 51:6 (1996), 1208-1209 (with K. A. Mirzoev).

[62] "Трехчленные рекуррентные соотношения с матричными коэффициентами. Вполне неопределенный случай”, Матем. заметки, 63:5 (1998), 709-716 (совм. с К.А. Мирзоевым); англ. пер.: "Three-term recurrence relations with matrix coefficients. The completely indefinite case", Math. Notes, 63:5 (1998), 624-630 (with K. A. Mirzoev).

[63] “Теория управления 'катастрофами'”, УМН, 53:3 (1998), 199-200 (совм. с Е. Д. Нурсултановым); англ. пер.: "Theory of control of 'catastrophes'", Russian Math. Surveys, 53:3 (1998), 628-629 (with E. D. Nursultanov).

[64] "Об устойчивости волчка с полостью, заполненной вязкой жидкостью", Функи. анализ и его прил., 32:2 (1998), 36-55 (совм. с А. А. Шкаликовым, М. Ю. Юркиным); англ. пер.: "On the stability of a top with a cavity filled with a viscous fluid", Funct. Anal. Appl., 32:2 (1998), 100-113 (with A. A. Shkalikov, M. Yu. Yurkin).

[65] "Обобщенные якобиевы матрицы и индексы дефекта обыкновенных дифференциальных операторов с полиномиальными коэффициентами", Функи. анализ и его прил., 33:1 (1999), 30-45 (совм. с К. А. Мирзоевым); англ. пер.: "Generalized Jacobi matrices and deficiency numbers of ordinary differential operators with polynomial coefficients", Funct. Anal. Appl., 33:1 (1999), 25-37 (with K. A. Mirzoev).

[66] “Задачи дифракции для периодической поверхности для уравнения Гельмгольца", Избранные вопросы математики и механики и их приложения, Изд-во МГУ, М., 1999 (совм. с А. А. Шкаликовым).

[67] "Бесконечномерные эллиптические координаты", Функи. анализ и его прил., 33:4 (1999), 73-78 (совм. с А. А. Степановым); англ. пер.: "Infinite-dimensional elliptic coordinates", Funct. Anal. Appl., 33:4 (1999), 300-303 (with A. A. Stepanov).

[68] "Об интегральных операторах в $L_{p}$-пространствах", Фундамент. и прикл. матем., 5:2 (1999), 475-491 (совм. с Е. Д. Нурсултановым).

[69] "Scattering of waves by periodic gratings and factorization problems", Recent advances in operator theory (Groningen, 1998), Oper. Theory Adv. Appl., 124, Birkhäuser, Basel, 2001, 361-383 (with A. A. Shkalikov).

[70] "Признаки вполне неопределенности якобиевых матриц с матричными коэффициентами", Функи. анализ и его прил., 35:4 (2001), 32-37 (совм. с К. А. Мирзоевым); англ. пер.: "Complete indefiniteness tests for Jacobi matrices with matrix entries", Funct. Anal. Appl., 35:4 (2001), 265-269 (with K. A. Mirzoev).

[71] "Задача об установившихся колебаниях полуцилиндра с кубической упругой структурой и связанные с ней самосопряженные квадратичные пучки", Докл. PAH, 400:3 (2005), 309-314 (совм. с М. Г. Аргета).

[72] "О спектре векторного оператора Шредингера", Докл. РАН, 411:4 (2006), 449-451 (совм. с Р. С. Исмагиловым); англ. пер.: "On the spectrum of a vector Schrödinger operator", 74:3 (2006), 854-856 (with R. S. Ismagilov). 
[73] "Об одной числовой характеристике пары симметрических операторов и спектре неполуограниченного векторного оператора Штурма-Лиувилля", Докл. РАН, 423:1 (2008), 19-21 (совм. с Р. С. Исмагиловым); англ. пер.: "An invariant of a pair of symmetric operators and the spectra of nonsemibounded Sturm-Liouville vector operators", 78:3 (2008), 854-856 (with R. S. Ismagilov).

[74] "О спектре векторного оператора Шрёдингера", Функи. анализ и его прил., 41:1 (2007), 39-51 (совм. с Р. С. Исмагиловым); англ. пер.: "On the spectrum of a vector Schrödinger operator", Funct. Anal. Appl., 41:1 (2007), 31-41 (with R. S. Ismagilov).

[75] "Об асимптотике спектра неполуограниченного векторного оператора ШтурмаЛиувилля”, Функи. анализ и его прил., 42:2 (2008), 11-22 (совм. с Р. С. Исмагиловым); англ. пер.: "On the asymptotics of the spectrum of a nonsemibounded vector Sturm-Liouville operator", Funct. Anal. Appl., 42:2 (2008), 89-97 (with R. S. Ismagilov).

[76] "О возмущении спектра дифференциального оператора, порожденном ограниченным изменением потенциала", Функи. анализ и его прил., 43:3 (2009), 54-64 (совм. с Р. С. Исмагиловым); англ. пер.: "Perturbation of the spectrum of a differential operator under a bounded perturbation of the potential", Funct. Anal. Appl., 43:3 (2009), 208-216 (with R. S. Ismagilov).

[77] "Спектральная асимптотика несамосопряженного дифференциального оператора с индефинитной весовой функцией”, Дифференц. уравнения, 45:4 (2009), 534-542; англ. пер.: "Spectral asymptotics of a nonselfadjoint elliptic differential operator with an indefinite weight function", Differ. Equ., 45:4 (2009), 549-557. 